\title{
Some Mathematics for Splashes: Sea-wave impact on coastal structures
}

\author{
Mark J. Cooker
}

\begin{abstract}
Structures built on the sea shore, such as harbour walls and breakwaters, are prone to damage by breaking waves. Such structures often need costly repairs especially after winter storms. The consulting company H.R. Wallingford gives advice to clients who design, build and repair seawalls. H.R. continually seek theories, models and simulations to predict the wave loads on coastal structures. Mathematics helps account for the surprisingly large forces exerted by sea waves hitting seawalls. A case is made for solving Laplace's equation, with mixed boundary conditions, to treat wave impact. Based on Euler's equations of fluid dynamics, the theory accounts for the high accelerations and pressures during the brief time of impact. We predict a sudden change in the water-velocity field in the impacting wave. Also there is an impulsive pressure field: the pressure-impulse is a useful concept and variable for an engineer to understand the loads on a structure when hit by a breaking wave. Solving mathematical problems can unveil the mystery and drama of breaking waves and splashes.
\end{abstract}

\section{Introduction}

Some of the most collaborative and creative times in my professional life have been spent while trying to solve companies' problems at European Study Groups with Industry. Typically, on the Monday of a week-long Study Group, the company presents a problem in physical terms, without any theory, and the question that the company wants answered may be unclear, even after days of debate. On the Friday each academic team gives a presentation to the company, after four days of wrestling with formulating it as a set of mathematical statements, and maybe a solution too! Always there are points for further mathematical exploration by the company. The

Dr M.J. Cooker

School of Mathematics, University of East Anglia, Norwich NR4 7TJ, UK m.cooker@uea.ac.uk 
company reps are usually delighted by the new ideas and progress made by the mathematicians.

The UK company Hydraulics Research Wallingford (H.R.) is an international industrial consultancy in areas such as ocean wave processes, flood risks, and the design of structures in marine environments. As long ago as 1990 I first presented my PhD research at a meeting at H.R. on wave forces. H.R.'s interest in my work comes from their ongoing wish to have more realistic theories and computer simulations to support the best engineering advice for clients designing or building coastal structures. A typical concern is, what influence will a new structure have on the wave regime at my site? Will the waves change, from just breaking benignly offshore, to breaking against the structure? And if breaking-wave impact does occur, what extreme forces might the structure have to withstand? See Fig. 1. In 1990 the standard engineering reference manuals said little about impact pressures and recommended testing laboratory-scale models of the structure. There were also peculiar observations at the sites of damaged structures - How can stone blocks in a seawall be sucked out by waves? How can rubble from a damaged breakwater move seawards?

In recent years H.R. have been interested in wave impact forces on, and inside complex structures. This is especially important when scaling-up measurements from laboratory models to make a forecast at full-scale. We will find that apart from the model equations, it is helpful to consider scaling the variables to identify dimensionless numbers. These constants also help classify problems.

In $\S 2$ and $\S 3$ we develop the theory and formulation of the governing equations and boundary conditions of Pressure-Impulse Theory. A solution relevant to the

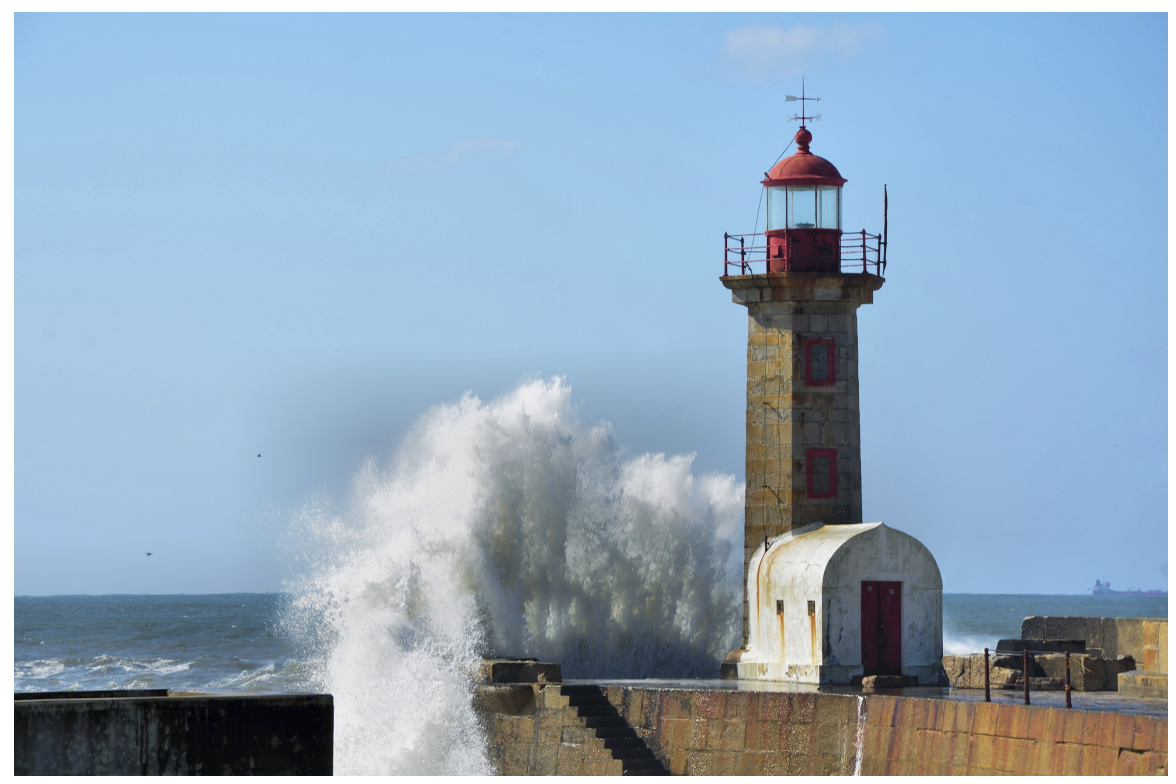

Fig. 1 Sea-wave impact. 
company's problem is worked out in $\S 4$. Lastly, $\S 5$ draws lessons from this chapter, and contains other topics that you can explore.

\section{Development of the Mathematical Model}

We follow the theory presented in [2] and [3]. We assume the wave water is frictionless (inviscid) and unsqueezable (incompressible) of fixed density $\rho=1000 \mathrm{~kg} / \mathrm{m}^{3}$. The fluid flow is described by a velocity vector field $\mathbf{v}(x, y, z, t)$, where $t$ is time and $x, y, z$ are a cartesian coordinate frame of reference fixed relative to the seawall and sea bed. The positive $x$-axis is horizontal and at right-angles to the shoreline. The positive $y$ axis points vertically up. For a plane seawall the $z$-axis runs horizontally along the seawall. From offshore, incoming waves can approach a seawall at any angle, but we focus on the most violent impacts which occur when the wave's forward motion is directed normal to the seawall. We have a two-dimensional problem in the $x, y$ plane. (The following theory is valid in three spatial dimensions.) Acheson [1] describes Euler's equations of fluid flow, in terms of the velocity and the gradient $\nabla$ of the pressure $p(x, y, t)$ :

$$
\frac{\partial \mathbf{v}}{\partial t}+(\mathbf{v} \cdot \nabla) \mathbf{v}=-\frac{1}{\rho} \nabla p-g \mathbf{j}
$$

where $g=9.81 \mathrm{~ms}^{-2}$ is the acceleration due to gravity. The wave water is incompressible so its velocity $\mathbf{v}=u \mathbf{i}+v \mathbf{j}+w \mathbf{k}$ has a constraint on its divergence $(\nabla \cdot)$ :

$$
\nabla \cdot \mathbf{v}=0 . \quad\left(\frac{\partial u}{\partial x}+\frac{\partial v}{\partial y}+\frac{\partial w}{\partial z}=0 .\right)
$$

Next we scale eq. (1) to find the dimensionless constants. The incident wave has a characteristic speed $U$, (e.g. the forward speed of the breaking wave face), and a characteristic length scale $h$, (e.g. water depth, or height of the breaking wave). The impact occurs in the time-interval $0 \leq t \leq \Delta t$, where we expect $\Delta t$ to be a very short time scale compared with $h / U$. We define an impact to be an event for which the dimensionless violence number $N_{v}=h /(U \Delta t)$, is large. We define dimensionless starred variables: $\mathbf{v}=U \mathbf{v}^{*}, x=h x^{*}, \quad y=h y^{*}, \quad z=h z^{*}, t=t^{*} \Delta t$ and $p=p_{0} p^{*}$, where the constant $p_{0}$ is chosen below. We substitute the new variables into eq. (1) and find that

$$
\frac{\partial \mathbf{v}^{*}}{\partial t^{*}}+\frac{U \Delta t}{h}\left(\mathbf{v}^{*} \cdot \nabla^{*}\right) \mathbf{v}^{*}=-\frac{p_{0} \Delta t}{\rho U h} \nabla^{*} p^{*}-\frac{g \Delta t}{U} \mathbf{j} .
$$

During an impact the fluid acceleration (whose magnitude is $U / \Delta t$ in our scaling) can be thousands of $g$. So the dimensionless number in the final term of (3), $g \Delta t / U$ is very much less than one. Hence we neglect the influence of gravity. Another dimensionless number, on the left of (3), is $U \Delta t / h=N_{v}^{-1}$, which is small, so the nonlinear term in eq. (3) is also negligible. We only have two terms left to balance 
the equation. The first term balances the pressure gradient provided the latter has coefficient equal to one. Consequently the pressure scaling is

$$
p_{0}=\frac{\rho U h}{\Delta t} .
$$

Equation (4) is important, as it helps us appreciate the huge pressure scale. Let's choose some consistent values: $U=10 \mathrm{~ms}^{-1}, h=10 \mathrm{~m}$ and a guess that $\Delta t=0.1 \mathrm{~s}$. Then $p_{0}=10^{6} \mathrm{Nm}^{-2}$, which is ten times atmospheric pressure.

We don't need to guess a value for $\Delta t$, or calculate $p_{0}$. The important theoretical and practical point is that the pressure-impulse, roughly the product $p_{0} \Delta t$, goes with a change in momentum. Equation (4) shows us that the scale of pressure-impulse is

$$
p_{0} \Delta t=\rho U h .
$$

In elementary mechanics, the impact of two snooker balls is treated using an impulse - a vector consisting of an indefinitely large contact force multiplied by an indefinitely small time $\Delta t$ of contact. We do not care about the sizes of the large force or the small time, because to compute the change in the two colliding balls' momenta we only need the product of the force and time, and the same is suggested by eq. (5). Next we show how to do impact mechanics for a fluid.

\section{Pressure-Impulse Theory}

Motivated by (5), first we define the pressure-impulse $P(x, y, z)$ :

$$
P(x, y, z)=\int_{0}^{\Delta t} p(x, y, z, t) \mathrm{dt} .
$$

We return to dimensional variables, and neglect all the terms in eq. (1), except for the first one on each side:

$$
\frac{\partial \mathbf{v}}{\partial t}=-\frac{1}{\rho} \nabla p
$$

Now we integrate with respect to time, $t \in[0, \Delta t]$ and substitute from (6). The lefthand side is transformed into the sudden change in velocity:

$$
\mathbf{v}_{\mathbf{a}}(x, y, z)-\mathbf{v}_{\mathbf{b}}(x, y, z)=-\rho^{-1} \nabla P(x, y, z),
$$

where $\mathbf{v}_{\mathbf{a}}$ is the velocity field at time $\Delta t$, just after impact, and $\mathbf{v}_{\mathbf{b}}$ is the velocity field at time $t=0$, just before impact. The subscripts $b$ for 'before', and $a$ for 'after', are used throughout. Equation (8) will be useful later for finding $\mathbf{v}_{\mathbf{a}}$ from $\mathbf{v}_{\mathbf{b}}$ after we have obtained the pressure-impulse, $P$.

The divergence of eq. (8) is zero, owing to eq. (2). So, in two space dimensions, eq. (8) implies that the pressure impulse $P$ satisfies Laplace's equation: 


$$
\frac{\partial^{2} P}{\partial x^{2}}+\frac{\partial^{2} P}{\partial y^{2}}=0
$$

The domain of eq. (9) is the specified fluid domain at $t=0$.

Next we derive the boundary conditions for (9). On the water surface, where the pressure is zero, eq. (6) ensures that $P=0$ there too. Next, suppose that part of the boundary of the fluid domain is a fixed impermeable surface, called B, with an outward normal unit vector $\mathbf{n}$. There are two possibilities:

(i) If fluid is in contact with $\mathrm{B}$ before impact then the impermeability condition ensures $\mathbf{n} \cdot \mathbf{v}_{\mathbf{a}}=0=\mathbf{n} \cdot \mathbf{v}_{\mathbf{b}}$. Hence the $\mathbf{n}$ component of eq. (8) implies $\mathbf{n} \cdot \nabla P=0$, and we write this boundary condition as $\partial P / \partial n=0$, for short. The sea bed is one place where this condition on boundary $\mathrm{B}$ applies.

(ii) A second possibility is, before impact the face of the wave approaches $\mathrm{B}$ with a non-zero normal velocity component $\mathbf{n} \cdot \mathbf{v}_{\mathbf{b}}$. After impact the fluid stays on B and flows tangentially to B. So $\mathbf{n} \cdot \mathbf{v}_{\mathbf{a}}=0$. Together these things imply that the $\mathbf{n}$ component of eq. (8) is $\mathbf{n} \cdot \nabla P=\rho \mathbf{n} \cdot \mathbf{v}_{\mathbf{b}}$, and we write this boundary condition as

$$
\partial P / \partial n=\rho \mathbf{n} \cdot \mathbf{v}_{\mathbf{b}}, \quad \text { on } \mathrm{B},
$$

on that part of the solid boundary B, that is struck by the fluid, and the RHS contains given data. The normal velocity component of a breaking wave face is close to the wave's phase speed, which in shallow water of depth $h$ is close to $\sqrt{g h}$. The boundary-value problem for $P=P(x, y, z)$ is now complete.

The problem is of mixed type in that $P=0$ is a Dirichlet condition, at the free surface, and $\partial P / \partial n$ given on $\mathrm{B}$, is a Neumann condition on the solid parts of the boundary. We next show what the theory can do with a simple solution of (9). It will tell us a bit about the fluid dynamics when an idealised wave hits a vertical wall.

\section{An Example Calculation with an Idealised Triangular Wave}

We remove as much as possible of the complexity of a real breaking wave, and try a geometry which is simple, but not too simple. We take a seawall that is vertical, a sea bed that is flat, and a free surface that is a sloping straight line, as in Fig. 2. The fluid domain is the interior of a right-angled triangle. The hypotenuse is the free surface, set at an angle to the horizontal of $\pi / 8$ radians $\left(22.5^{\circ}\right)$. The sea bed is the $x$-axis $(y=0)$ between $x=0$ and $x=a$, where $a>0$ is a given constant. The vertical wall lies at $x=a$, from $y=0$ up to $y=h$, where $h$ is the water depth at the wall $h=a \tan (\pi / 8)=(\sqrt{2}-1) a=0.4142 a$.

On the bed the normal derivative $\partial P / \partial y=0$, and on the wall we suppose that the wave face just before impact has a component in the direction $\mathbf{n}=\mathbf{i}$, normal to the wall, of $U\left(1-3 y^{2} / a^{2}\right)$, where $U$ is a prescribed constant equal to the normal component of velocity at the foot of the wall. From eq. (10) we find 


$$
\frac{\partial P}{\partial x}=\rho U\left(1-\frac{3 y^{2}}{a^{2}}\right) \quad 0 \leq y \leq h .
$$

We model $U=\sqrt{g h}$, where $h$ is the water depth at the wall. This boundary data is crafted to fit a simple solution of Laplace's eq. (9). It describes a wave whose forward face is in most violent impact with the foot of the wall, and smallest impact velocity component $(0.515 \mathrm{U})$ at the top of the wall. (General boundary data is treated in [3], [4].) We must also have $P=0$ on the free surface, $y=(\sqrt{2}-1) x$.

A quick way to solve Laplace's eq. (9) comes from the fact that the real (imaginary) part of an analytic function of a complex variable $x+i y$ is a solution. E.g. a real constant $k$ times $\operatorname{Re}\left\{(x+i y)^{4}\right\}$. After fixing the value of $k$ to satisfy eq. (11), the pressure-impulse is

$$
P(x, y)=(\rho U h) \frac{1}{4 a^{3} h}\left(x^{4}-6 x^{2} y^{2}+y^{4}\right) .
$$

You can check: $\partial P / \partial y=0$ on the bed at $y=0$, and $P=0$ on $y=(\sqrt{2}-1) x$.

Next we discuss the consequences of eq. (12). The contours of constant $P(x, y)$ are drawn in Fig. 3. The pressure-impulse has its global maximum value of $0.604 \rho U h$ at the foot of the wall. As we go up the wall, $P$ decreases to zero at the top, where the free surface meets the wall.

The pressure-impulse at a point in the fluid can be converted to a pressure maximum with respect to time, from eq. (4). More useful to an engineer is the total impulse $I$, exerted by the wave on the wall (per unit length of wall): $I$ is the integral of $P$ from $y=0$ to $y=h$, at $x=a$ :

$$
I=0.4 \rho U h^{2}
$$

Formula (13) has a factor 0.4 - a pure number that depends on the shape of the wave. Also $I$ is directly proportional to density $\rho$ and the impact speed $U$. More interestingly, $I$ increases as the square of the height $h$ of the wave - if the wave is 3 times higher it delivers 9 times the punch! E.g. if $h=5 \mathrm{~m}$ and $U=7 \mathrm{~m} / \mathrm{s}$, then $I=70,000 \mathrm{Ns}$ per metre length of wall. This is the same impulse delivered by a 5 -tonne truck in a collision at 30 miles per hour.

The velocity field just after impact $\mathbf{v}_{\mathbf{a}}$, is found from (8) in which we now know $P(x, y)$. We choose the water velocity just before impact $\mathbf{v}_{\mathbf{b}}$, to be horizontal and given. E.g. $\quad \mathbf{v}_{\mathbf{b}}=U\left(1-3 y^{2} a^{-2}\right) \mathbf{i}$. Here $\mathbf{v}_{\mathbf{b}}$ is consistent with eq. (11). Taking the gradient of expression (12), the velocity field just after impact is

$$
\mathbf{v}_{\mathbf{a}}(x, y)=\frac{U}{a^{3}}\left(\left[a^{3}-3 a y^{2}-x^{3}+3 x y^{2}\right] \mathbf{i}+y\left[3 x^{2}-y^{2}\right] \mathbf{j}\right) .
$$

We may evaluate this velocity anywhere in the fluid domain of Fig. 2 . The velocity at the free surface is obtained by setting $y=x h / a=(\sqrt{2}-1) x=0.4142 x$. For $x: 0 \leq x \leq a$ the velocity after impact is 


$$
\mathbf{v}_{\mathbf{a}}(x, h x / a)=U\left(\left[1-0.5147 \frac{x^{2}}{a^{2}}-0.4853 \frac{x^{3}}{a^{3}}\right] \mathbf{i}+1.172 \frac{x^{3}}{a^{3}} \mathbf{j}\right) .
$$

The upward, $\mathbf{j}$, component is greatest at $x=a$, where the free surface meets the wall, and the maximum is $1.17 \mathrm{U}$. This is 2.4 times greater than the fluid's speed at this point just before impact. The velocity (15) describes the beginning of a jet, that splashes up the wall. Equation (15) implies that after a short time $\Delta t$, the free surface is near to $y=0.4142 x+1.172 U \Delta t x^{3} / a^{3}$.

Mathematical modelling shows what waves can do to a structure in terms that are qualitative (what type?) and quantitative (how much?). This is the kind of theoretical tool that H.R. seeks. Section 10 of [5] discusses the company's use of the theory for a harbour wall.

\section{Conclusions and Further Explorations}

When the violence number $N_{v}=h /(U \Delta t)$ is large, Pressure-Impulse Theory can be used to find the sudden change in wave velocity. When $N_{v}$ is large the gradient of pressure-impulse balances the abrupt change in fluid momentum - snooker-ball mechanics for fluids. Like the idea of impulse in particle mechanics, the pressureimpulse $P(x, y, z)$ is easier to handle than pressure, $p(x, y, z, t)$. Another advantage is that the domain is the initial position of the fluid domain. The pressure impulse obeys Laplace's equation, subject to mixed conditions. Once $P$ has been found we can then find the velocity just after impact and the total impulse on the wall. The pressure-impulse theory is flexible. It estimates what waves can do at a particular site, either to an existing structure or one in design. These insights aim to help H.R. in discussions, computations and measurements.

Topics that you can investigate include the following. First, evaluate the impulsive moment about the foot of the wall. Secondly, find the volume of water that an impact throws upwards and forwards over the seawall. (H.R. has an ongoing interest in overtopping as it is a hazard to pedestrians and vehicles parked on top of harbour walls.) Thirdly, model the fate of debris lost from damaged breakwaters. In Fig. 3 notice the horizontal gradient of pressure-impulse along the sea bed, from the maximum of $P$ at the wall towards lower values of $P$ near the origin. The fluid impulse on a boulder on the bed is modelled in [2], [4]. Another topic of interest is when the seawall is a row of caissons. Each caisson (box) moves due to an impulse, and it slides until friction brings it to a stop. The displacement is proportional to the square of the impulse.

You might try using Pressure-Impulse Theory in other situations. I have supervised project investigations of the skipping of stones thrown onto a water surface, in the ancient game of Ducks \& Drakes. The theory accounts for the repeated bouncing of the stone - or of cannon balls achieved in 18th-century naval gunnery - a trick that inspired Sir Barnes Wallis for his 'bouncing bomb'! 


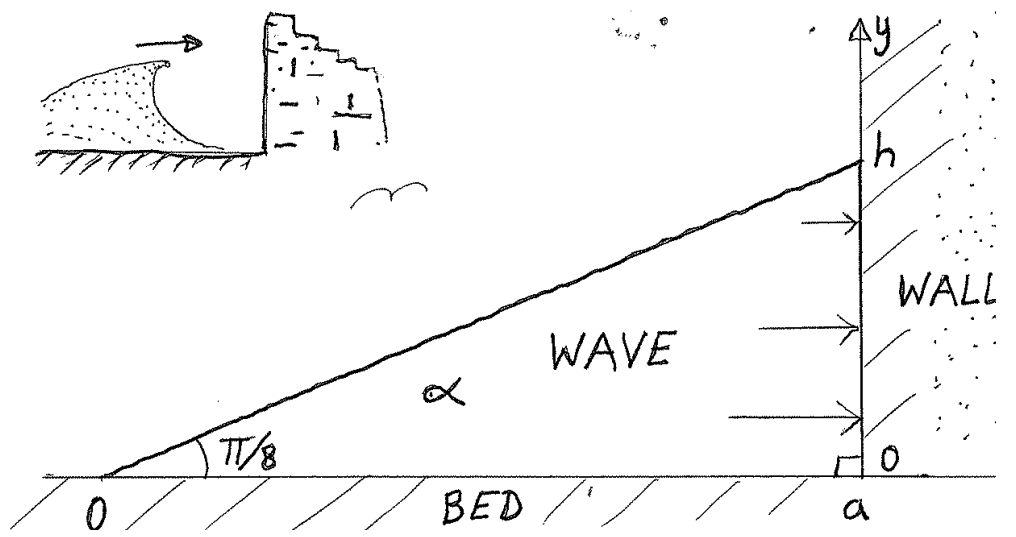

Fig. 2 Upper left: sketch of an approaching wave. Main: an idealised triangular wave at the start of its impact against a vertical wall. The incident velocity field is prescribed so that the face of the wave hits the wall at $x=a$.

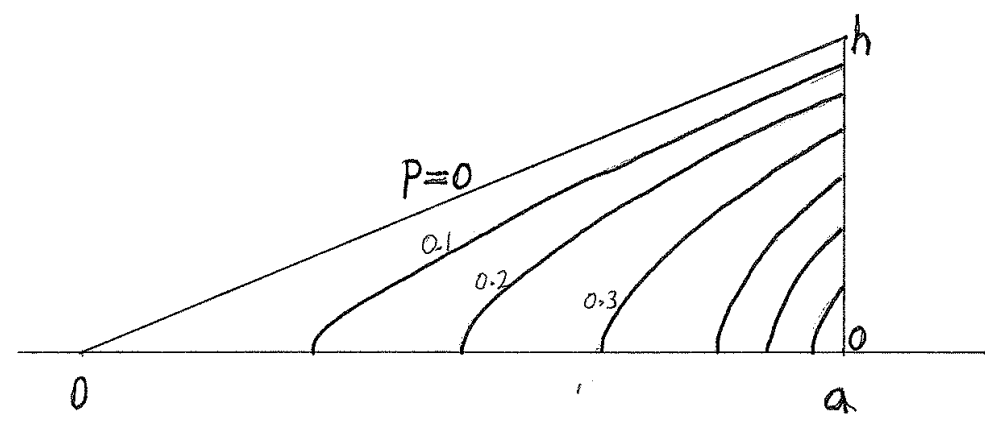

Fig. 3 Contours of constant pressure-impulse $P$, in units of $\rho U h$. The free-surface (hypotenuse) is $P=0$. The maximum $P=0.604 \rho U h$, occurs at the foot of the wall.

\section{References}

1. ACheson, D.J. 1990 Elementary Fluid Dynamics. Oxford University Press.

2. CoOKer, M.J. \& Peregrine, D.H. 1992 Wave impact pressure and its effect upon bodies lying on the sea bed. Coastal Engineering 18, 205-229.

3. Cooker, M.J. \& Peregrine, D.H. 1995 Pressure-impulse theory for liquid impact problems. Journal of Fluid Mechanics 297, 193-214.

4. CoX, S.J. \& COOKER, M.J. 1999 The motion of a rigid body impelled by sea-wave impact. Applied Ocean Research 21, 113-125.

5. Cuomo, G., Allsop, W., Bruce, T. \& Pearson, J. 2010 Breaking wave loads at vertical seawalls and breakwaters. Coastal Engineering 57(4), 424-439. 